\title{
Animal Sources
}

National Cancer Institute

\section{Source}

National Cancer Institute. Animal Sources. NCI Thesaurus. Code C18634.

Indicates that a product is derived from an animal. 